\title{
A fluorescence lifetime-based fibre-optic glucose sensor using glucose/ galactose-binding protein
}

\author{
Tania Saxl, ${ }^{* a}$ Faaizah Khan, ${ }^{a}$ Matteo Ferla, ${ }^{a}$ David Birch $^{b}$ and John Pickup ${ }^{a}$ \\ Received 21st June 2010, Accepted 24th November 2010 \\ DOI: 10.1039/c0an00430h
}

\begin{abstract}
Alternative, non-electrochemistry-based technologies for continuous glucose monitoring are needed for eventual use in diabetes mellitus. As part of a programme investigating fluorescent glucose sensors, we have developed fibre-optic biosensors using glucose/galactose binding protein (GBP) labelled with the environmentally sensitive fluorophore, Badan. GBP-Badan was attached via an oligohistidine-tag to the surface of Ni-nitrilotriacetic acid (NTA)-functionalized agarose or polystyrene beads.

Fluorescence lifetime increased in response to glucose, observed by fluorescence lifetime imaging microscopy of the GBP-Badan-beads. Either GBP-Badan agarose or polystyrene beads were loaded into a porous chamber at the end of a multimode optical fibre. Fluorescence lifetime responses were recorded using pulsed laser excitation, high speed photodiode detection and time-correlated singlephoton counting. The maximal response was at $100 \mathrm{mM}$ glucose with an apparent $K_{\mathrm{d}}$ of $13 \mathrm{mM}$ (agarose) and $20 \mathrm{mM}$ (polystyrene), and good working-day stability was demonstrated. We conclude that fluorescence lifetime fibre-optic glucose sensors based on GBP-Badan are suitable for development as clinical glucose monitors.
\end{abstract}

\section{Introduction}

The current sensors for continuous glucose monitoring in diabetes which are used in clinical practice are subcutaneously implanted needle-type enzyme electrodes or microdialysis probes. Both are based on glucose oxidase and either the electrochemical detection of hydrogen peroxide produced by the oxidation of glucose or 'wired' electron transfer from the enzyme to an underlying electrode. ${ }^{1}$ Such glucose sensors are making a useful contribution to the management of diabetic patients ${ }^{2}$ but have suboptimal accuracy and performance, due to impaired responses in vivo and signal drift. This necessitates frequent calibration against capillary blood glucose samples. ${ }^{3}$ The possible reasons for this inaccuracy include interfering electroactive substances in the tissues, coating of the sensor by protein and cells and changes in blood flow that alter oxygen access. $^{3}$ Alternative sensing technologies not based on electrochemistry or glucose oxidase are therefore being actively investigated.

Fluorescence-based glucose sensing is being researched by several groups ${ }^{4,5}$ and has the advantages of sensitivity and lack of electrochemical interference. Moreover, measurement of fluorescence decay lifetime, as opposed to intensity, has special potential for in vivo sensing ${ }^{6,7}$ because it is relatively independent

aDiabetes Research Group, King's College London School of Medicine, Guy's Hospital, London, SE1 1UL, UK

${ }^{b}$ Dept of Physics, SUPA, University of Strathclyde, Glasgow, G4 ONG, UK of light scattering in the tissues, signal amplitude fluctuations and fluorophore concentration - thus coating of an implanted sensor, which may reduce fluorescence intensity, will leave the lifetime unaltered.

Fluorescence glucose sensors may be envisaged as totally implanted, ${ }^{8}$ formulated as microcapsules for impregnation in the skin as a 'smart tattoo', ${ }^{9-12}$ as nanocapsules (pebbles or polymersomes) for intracellular sensing, ${ }^{13,14}$ as an intravenous catheter, ${ }^{15}$ and as a fibre-optic probe. ${ }^{16-19}$ Fibre-optic glucose sensors have the attraction of ease of implantation into and removal from the subcutaneous tissue, containment of the sensing element, and communication between the tissues and instrumentation outside the body via a waveguide which is minimally invasive, electrical isolated and generally biocompatible. ${ }^{\mathbf{1 8 , 2 0}}$

Several receptors for glucose have been investigated for fluorescence-based glucose sensing ${ }^{4}$ including boronic acid derivatives, ${ }^{4}$ concanavalin $\mathrm{A}^{4,17,19,21}$ and other lectins, ${ }^{8}$ glucose oxidase $^{4,13,16}$ and bacterial glucose/galactose-binding protein (GBP). ${ }^{122-30}$ GBP undergoes a large change in molecular conformation on binding glucose, which can be detected by either fluorescence resonance energy transfer or by labelling with an environmentally sensitive fluorophore. ${ }^{12,29,30}$ The binding constant for native GBP is in the micromolar range and therefore unsuitable for clinical glucose monitoring, but we recently reported $^{30}$ the synthesis of a new triple mutant of GBP with a $K_{\mathrm{d}}$ of $11 \mathrm{mM}$ and operating range of up to $100 \mathrm{mM}$ glucose, which we labelled with the environmentally sensitive fluorophore, 
Badan, at a position near the binding site. Addition of glucose produced a large increase in both fluorescence intensity and lifetime. The responses to glucose of the GBP-Badan were the same in serum and buffer. ${ }^{30}$

We now report the use of the high- $K_{\mathrm{d}}$ mutant of GBP (labelled with Badan) in a fibre-optic glucose sensor and proof-of-concept testing in vitro. A significant barrier to the use of GBP in such devices is immobilization of the protein on the probe without impairment of the conformation-based sensing. Our preliminary research (M. Ferla and J. C. Pickup, unpublished studies) indicated that several commonly used immobilization strategies such as entrapment in silica sol-gels and covalent linking to hydrogels can impair glucose sensing. We therefore investigated and report here the use of binding of GBP-Badan to Ni-nitrilotriacetic acid (NTA)-functionalised agarose or polystyrene beads, via an engineered oligohistidine tag on the GBP, and the employment of this immobilized protein in a fibre-optic glucose sensor.

The technology for fluorescence lifetime measurement is now well-established. ${ }^{7}$ Although fluorescence lifetime is used less in sensors than in spectroscopy and microscopy, optical fibrecoupled fluorescence lifetime measurements have been proven for some time. ${ }^{31}$ Indeed the proof of principle is already established for lab-on-a-chip distributed multiplexed sensor networks whereby several metabolites could be monitored simultaneously for point-of-care applications. ${ }^{32}$

Here we have used time domain pulsed optical excitation, but the same glucose sensor would be equally compatible with frequency-domain phase-modulation methods. ${ }^{6}$

\section{Results and discussion}

\section{Ni-NTA agarose beads for binding GBP-Badan}

Ni-NTA functionalised agarose beads were linked to His-tagged GBP-Badan as the glucose receptor and imaged using epi-fluorescence microscopy. The beads, in glucose-free phosphatebuffered saline (PBS), displayed high fluorescence intensity due to the large amount of protein bound to the Ni-NTA agarose (Fig. 1a).

Two fixed fluorescence lifetimes for GBP-Badan linked to agarose were generated from a global bi-exponential fit to the data. These were found to be 2.7 and $0.9 \mathrm{~ns}$, which from previous work may be considered to correspond respectively to the glucose-bound (closed) and unbound (open) configurations of GBP-Badan. ${ }^{12}$ These lifetimes differ only slightly from previously measured values for GBP-Badan in solution, most likely due to the immobilisation of the protein.

On addition of glucose, changes are observed in the fractional intensity $\left(F_{\mathrm{i}}\right)$ of each lifetime $\left(\tau_{\mathrm{i}}\right)$. For a bi-exponential decay, a mean lifetime $(\tau)$ is derived from the following equation:

$$
\tau=F_{1} \tau_{1}+F_{2} \tau_{2}
$$

where

$$
F_{1}+F_{2}=1
$$

Fluorescence lifetime images in glucose-free buffer and saturating $(100 \mathrm{mM})$ glucose showed an increase in the mean lifetime with addition of glucose (Fig. $1 \mathrm{~b}$ and c).

This fluorescence response is similar to our previous work on mutants of GBP labelled with Badan ${ }^{29,30}$ and studied in solution and in vesicles, where an increase in fluorescence intensity and lifetime was found with glucose addition. The present studies confirm that the response to glucose was retained when GBPBadan was bound to the agarose beads.

The main use of Ni-NTA bound to a solid matrix such as agarose beads to date has been in 'immobilized metal-affinity chromatography', i.e. the purification of recombinant proteins, where a genetically engineered polyhistidine tag on one terminus of the protein binds to the support via a metal-histidine interaction. ${ }^{33}$ NTA has four chelating sites, allowing two nickel coordination sites free to bind to the protein. To the best of our knowledge, Ni-NTA-His tag binding has not previously been used to immobilize protein at fibre-optic biosensors.

\section{Ni-NTA functionalized agarose beads for use in a fibre-optic glucose sensor}

The dose-response curve for the GBP-Badan-agarose beads incorporated in a fibre-optic glucose sensor is shown in Fig. 2. The percentage change in the mean fluorescence lifetime is

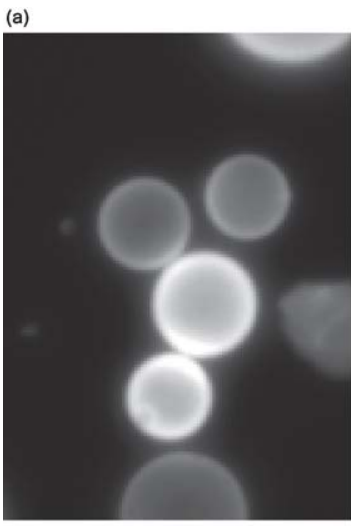

(b)

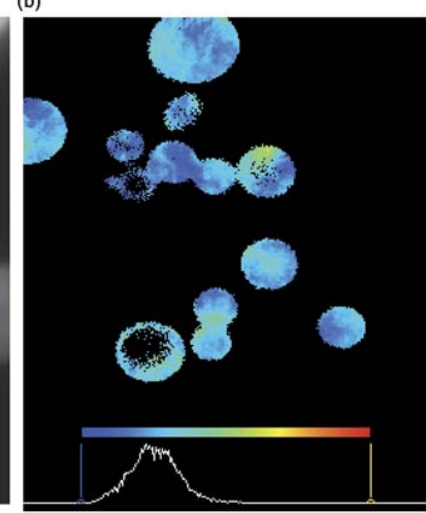

Lifetime / ns $\quad 3$

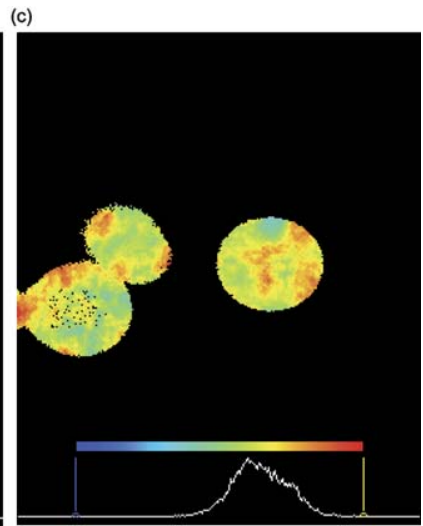

Lifetime / ns

3

Fig. 1 Ni-NTA agarose beads with bound GBP-Badan. (a) Epi-fluorescence intensity image $\left(\lambda_{\mathrm{ex}}=400 \mathrm{~nm}\right.$ and $\lambda_{\mathrm{em}}=550 \mathrm{~nm}$ ). Mean fluorescence lifetime images of beads in $10 \mathrm{mM}$ PBS and (b) zero glucose, and (c) saturated (100 mM) glucose. 


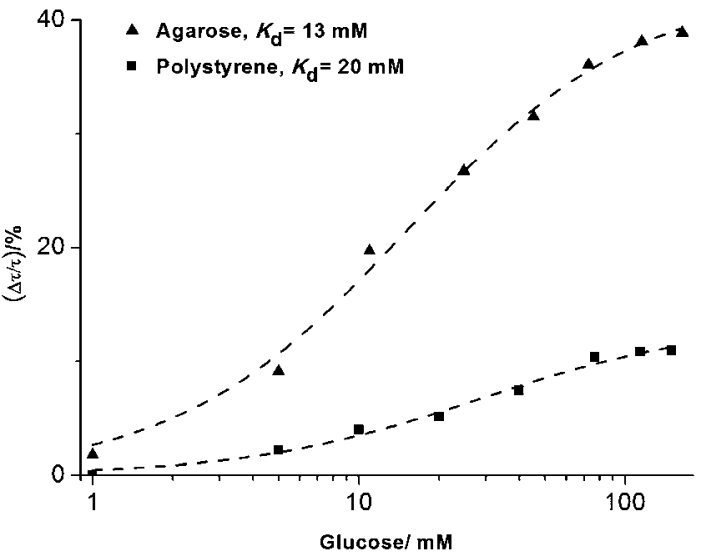

Fig. 2 Percent change in mean fluorescence lifetime against glucose concentration, for Ni-NTA agarose and Ni-NTA polystyrene-based glucose sensors. $K_{\mathrm{d}}$ values were estimated by fitting with a hyperbolic dose-response function.

plotted, corresponding to a change of $0.6 \mathrm{~ns}$ over the measured glucose range. The apparent $K_{\mathrm{d}}$ was estimated at $13 \mathrm{mM}$ from a hyperbolic fit to the data, and is thus suitable for glucose measurement over the physiological and pathophysiological range. This compares with our previously reported $K_{\mathrm{d}}$ of $11 \mathrm{mM}$ for this mutant of GBP labelled with Badan but when studied in solution. ${ }^{30}$ However, the apparent $K_{\mathrm{d}}$ of the sensor represents the operating range of the device, rather than the true binding constant of the GBP-Badan.

The response time from 0 to $100 \mathrm{mM}$ glucose concentration was $10 \mathrm{~min}$ (Fig. 3), with a reversible response on removal of glucose, although the 'off' response time increased to approximately one hour.

\section{Fibre-optic glucose sensor with GBP-Badan bound to Ni-NTA functionalized polystyrene beads}

Individual lifetime values of $2.7 \mathrm{~ns}$ and $0.8 \mathrm{~ns}$ were generated by the bi-exponential fit for GBP-Badan bound to polystyrene

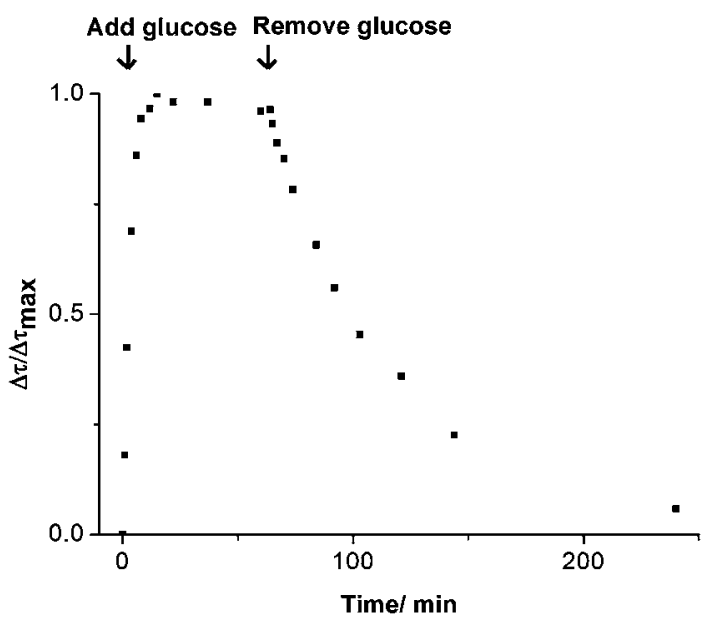

Fig. 3 Time course for the agarose bead fibre-optic sensor, for addition and removal of saturating glucose (expressed as fraction of the maximal response). beads, similar to the lifetime values for agarose-bound GBPBadan.

The dose-response curve for the fibre-optic glucose sensor based on GBP-Badan linked to Ni-NTA polystyrene beads also showed an increase in mean fluorescence lifetime on addition of glucose, though with a smaller maximal increase corresponding to $0.25 \mathrm{~ns}$ (Fig. 2). The $K_{\mathrm{d}}$ was estimated at $20 \mathrm{mM}$ from a hyperbolic fit to the data.

The response time of the polystyrene bead-based sensor from 5 to $20 \mathrm{mM}$ glucose was approximately $10 \mathrm{~min}$, with an 'off' response time similar to that of the agarose sensor. There was good stability over the course of a working day (Fig. 4). Fluorescence lifetime is considered to be independent of photobleaching. As the sensor response was found to be stable throughout the course of the day in our study, it can be concluded that any photo-bleaching that occurred had no effect on the signal, and the theoretical advantage of lifetime-based sensing over intensity-based sensing is confirmed.

\section{Experimental}

\section{Reagents and materials}

PBS (10 mM, pH 7.4) was used unless otherwise stated. The triple mutant of GBP (H152C/A213R/L238S) was synthesized and labelled with the environmentally sensitive fluorophore, Badan (Invitrogen, Paisley, UK), covalently linked at position 152C, as previously described. ${ }^{30}$ The protein was engineered with a hexahistidine tag at the $C$-terminus, which enabled binding to the $\mathrm{Ni}-$ NTA moiety. Ni-NTA agarose beads (100-200 $\mu \mathrm{m}$ diameter) were obtained from Qiagen (Crawley, UK). Ni-NTA polystyrene beads (100 $\mu \mathrm{m}$ diameter) were obtained from Micromod (Rostock, Germany). 0.48 NA, hard polymer-clad multimode optical fibre (high $\mathrm{OH}, 1000 \mu \mathrm{m}$ core) was from Thorlabs (Ely, UK). Hydrophilic nylon mesh ( $20 \mu \mathrm{m}$ pore size) was obtained from Spectrum Labs (Rancho Dominguez, CA, USA).

\section{Attachment of GBP-Badan to agarose or polystyrene beads}

$5 \mu \mathrm{l}$ packed Ni-NTA agarose beads were washed three times in PBS to remove ethanol and the supernatant removed. Beads were

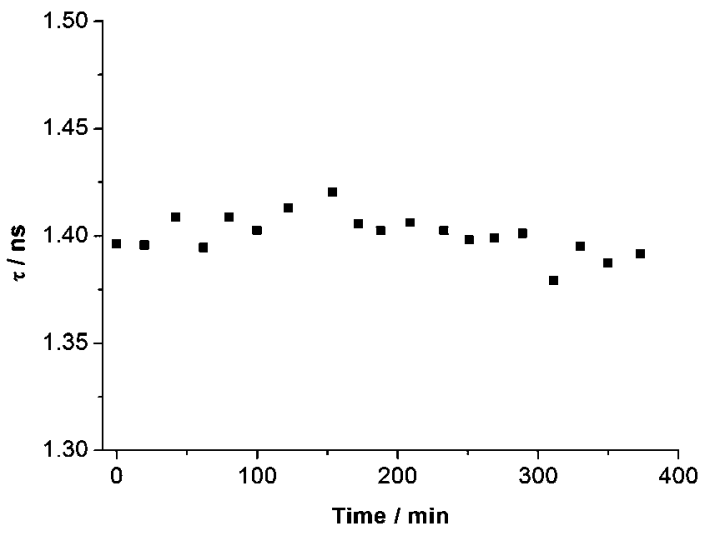

Fig. 4 Stability of the Ni-NTA polystyrene bead-based fibre-optic glucose sensor over the course of a working day. The scale on the $y$-axis is reflective of the range of the sensor, from the minimum to maximum lifetime. 
then incubated with $20 \mu \mathrm{l}$ of GBP-Badan $(50 \mu \mathrm{M})$ in PBS for 4 hours on a roller at $25^{\circ} \mathrm{C}$. The beads were washed again and finally re-suspended in $10 \mu \mathrm{l}$ PBS. Ni-NTA functionalised polystyrene beads ( $20 \mu \mathrm{l}$ of $50 \mathrm{mg} \mathrm{ml}^{-1}$ solution) were labelled with GBP-Badan in a similar fashion.

\section{Construction of fibre-optic glucose sensor}

The surface of $1000 \mu \mathrm{m}$ core multimode fibre (Thorlabs) was prepared by controlled breaking using a diamond scribe. A $2 \mathrm{~mm}$ long chamber for the glucose-sensing agarose or polystyrene beads was formed using a section of $1.5 \mathrm{~mm}$ diameter glass tubing and secured with epoxy resin at the tip of the fibre optic, as illustrated in Fig. 5. The beads were placed in the chamber and a wetted $3 \mathrm{~mm}$ circle of nylon mesh was applied to the end of the fibre, covering the chamber, and fixed in place using a rubber $\mathrm{O}-$ ring.

\section{Fluorescence lifetime measurement with fibre-optic glucose sensor}

The experimental set-up for fluorescence excitation and recording is shown in Fig. 6. Excitation was provided by a supercontinuum, femto second pulsed laser (sc400, Fianium, Southampton, UK) filtered to $417 \pm 30 \mathrm{~nm}$. Emission was filtered and collected at $542 \pm 25 \mathrm{~nm}$ using a high speed photomultiplier tube (PMT) (PMH-100, Becker and Hick1, Berlin, Germany). The signal from the PMT was processed using a timecorrelated single-photon counting (TCSPC) card (spc830, Becker and Hick1). Data were analysed using TRI2 software, courtesy of Paul Barber, Grey Cancer Institute, Oxford and as previously described. ${ }^{12}$ Mean lifetimes were calculated from the fractional intensities, generated by a bi-exponential fit as previously described. ${ }^{12}$

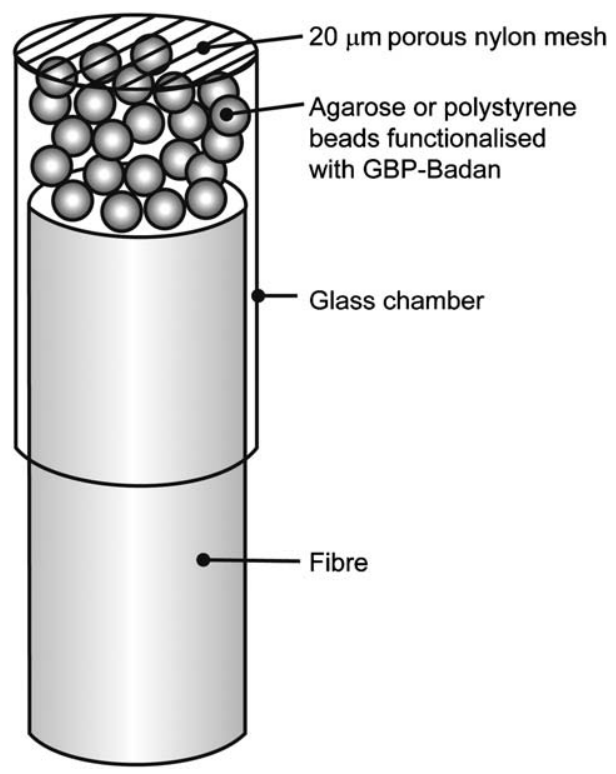

Fig. 5 Diagram of the terminus of the fibre-optic glucose sensor containing GBP-Badan labelled beads.

\section{Lens}

Band pass and razor edge filters

Beam splitter

Condenser lens

Fibre

positioner

Fibre
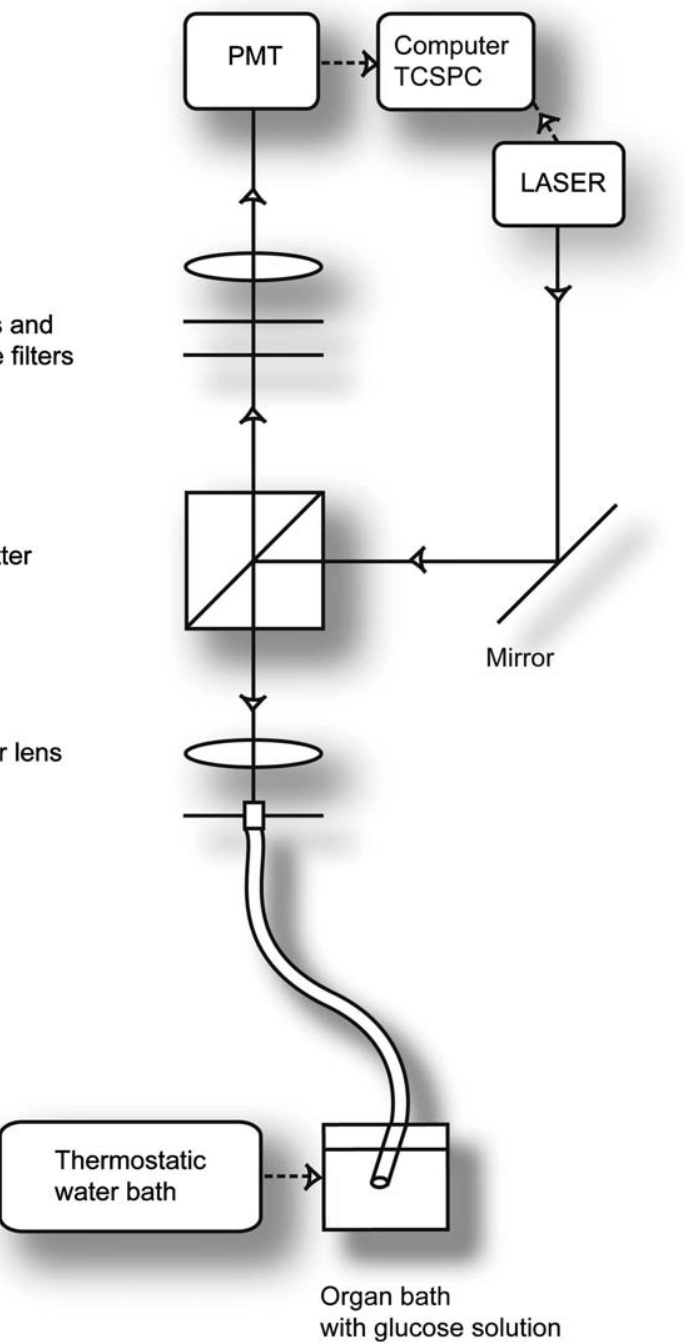

Fig. 6 Diagram of the experimental set up. A femto-second pulsed, supercontinuum laser was filtered to $417 \mathrm{~nm}$ and imaged at the terminus of a $1000 \mu \mathrm{m}$ multi-mode fibre. Fluorescence from the sensor terminus of the fibre was collected, filtered and laser excitation removed using a 540 $\mathrm{nm}$ band pass filter and $495 \mathrm{~nm}$ razor edge filter. Fluorescent emission was acquired by a high-speed PMT and processed by time-correlated single-photon counting (TCSPC) electronics.

\section{Fluorescence intensity and lifetime imaging microscopy}

GBP-Badan bound to agarose or polystyrene beads was imaged using an upright 90i fluorescence microscope (Nikon, Tokyo, Japan) and custom-built fluorescence lifetime imaging instrumentation, as previously described. ${ }^{12}$

\section{Glucose sensor responses}

For in vitro testing, the fibre-optic glucose sensor was immersed in $100 \mathrm{ml}$ PBS in a jacketed glass organ bath, with constant stirring and temperature maintained at $26^{\circ} \mathrm{C}$ using a thermostatically controlled water bath (GD120, Grant Instruments, Shepreth, UK). Aliquots of $1 \mathrm{M}$ stock glucose solution were added sequentially to alter the glucose concentration. 
For stability experiments, the optical fibre was immersed in 20 $\mathrm{mM}$ glucose solution in an organ bath as before, and maintained at $25.5-25.9^{\circ} \mathrm{C}$ with stirring.

\section{Conclusion}

These studies demonstrate proof-of-concept for a fibre-optic glucose sensor using GBP-Badan and measuring fluorescence lifetime changes. The sensor response was best conserved using Ni-NTA functionalised agarose beads as an immobilisation strategy. Ni-NTA functionalised polystyrene beads result in a slightly reduced response (percent change in fluorescence lifetime), possibly due to charge effects that alter the fluorescence of the Badan.

Glucose responses of the immobilised sensor are reversible and stable and would allow clinical measurements about every one hour. Although this would be of significant clinical use, decreasing the response time, particularly the 'off' response, would be valuable for monitoring rapidly changing blood glucose concentrations. One strategy for accomplishing this may be miniaturization of the fibre-optic probe (which in any case will be needed for in vivo use), since previous work by others has shown much faster response times with highly miniaturized fluorescence glucose optodes. ${ }^{16}$

The realistic clinical value of a glucose sensor based on our system will depend on demonstration of in vivo reversibility, biocompatibility and lack of interference, and for a wearable device, a miniaturised fluorescence lifetime measurement system must be developed. The most important interfering substance which binds to GBP is galactose, but in the concentration present in plasma, this is unlikely to be a major problem in clinical studies. The sensor was characterised using a pulsed laser and time-correlated single-photon counting, however, a less complex and potentially wearable system for clinical use should be achievable. Indeed optical fibre coupling to a single integrated circuit comprising light emitting ${ }^{34}$ or laser diode excitation, avalanche photodiode detection and data processing in either the time or frequency domain is now possible.

We demonstrate for the first time here that Ni-NTA linked to solid supports such as agarose can be used to immobilize GBP for use in a fibre-optic biosensor, and that this immobilization does not impair the fluorescence response to glucose seen previously in solution ${ }^{29,30}$ and in microcapsules. ${ }^{12}$ Other recombinant proteins with an oligohistidine tag might also be immobilized in this way at biosensor probes.

Following this proof-of-concept of a GBP-based fibre-optic glucose sensor in vitro, the way is now clear for in vivo studies, say with the probe implanted in the subcutaneous tissue, as a preliminary to clinical exploitation as a continuous glucose monitor.

\section{Acknowledgements}

We are grateful to EPSRC for a Science and Innovation Award and the Diabetes Foundation for additional financial support. We thank Dr D. Matthews and Dr S. Ameer-Beg (King's College
London, London, UK) for assistance with the construction of the fluorescence lifetime instrumentation.

\section{References}

1 D. C. Klonoff, Diabetes Care, 2005, 28, 1231-1239.

2 Juvenile Diabetes Research Foundation Continuous Glucose Monitoring Study Group, N. Engl. J. Med., 2008, 359, 1464-1476.

3 J. C. Pickup, F. Hussain, N. D. Evans and N. Sachedina, Biosens. Bioelectron., 2005, 20, 1897-1902.

4 J. C. Pickup, F. Hussain, N. D. Evans, O. J. Rolinski and D. J. S. Birch, Biosens. Bioelectron., 2005, 20, 2555-2565.

5 E. A. Moschou, B. V. Sharman, S. K. Deo and S. Daunert, Fluorescence glucose detection: advances toward the ideal in vivo biosensor, J. Fluoresc., 2004, 14, 535-547.

6 J. R. Lakowicz, Principles of Fluorescence Spectroscopy, Plenum Press, New York, 2nd edn, 1999.

7 D. J. S. Birch and R. E. Imhof, in Topics in Fluorescence Spectroscopy, ed. J. R. Lakowicz, Plenum Press, New York, 1991, vol. 1, pp. 1-95.

8 J. K. Nielsen, J. S. Christiansen, J. S. Kristensen, H. O. Toft, L. L. Hansen, S. Aasmaul and K. Gregorius, J. Diabetes Sci. Technol., 2009, 3, 98-109.

9 S. Chinnayelka and M. J. McShane, Diabetes Technol. Ther., 2006, 8, 269-278.

10 J. C. Pickup, Z.-L. Zhi, F. Khan, T. Saxl and D. J. S. Birch, Diabetes/ Metab. Res. Rev., 2008, 24, 604-610.

11 A. Chaudhary and M. Raina, et al., Conf. Proc. IEEE Eng. Med. Biol. Soc., 2009, 1, 4098-40101.

12 T. Saxl, F. Khan, D. R. Matthews, Z.-L. Zhi, O. Rolinski, S. AmeerBeg and J. C. Pickup, Biosens. Bioelectron., 2009, 24, 3229-3234.

13 H. Xu, J. W. Aylott and R. Kopelman, Analyst, 2002, 127, 1471-1477.

14 C. LoPresti, H. Lomas, M. Massignani, T. Smart and G. Battaglia, J. Mater. Chem., 2009, 19, 3576-3590.

15 Glumetrics, Presented at 3rd International Conference on Advanced Technologies and Treatments for Diabetes, Basel, February 2010.

16 Z. Rosenweig and R. Kopelman, Anal. Chem., 1996, 68, 1408-1413.

17 K. C. Liao and T. Hogen-Esch, et al., Biosens. Bioelectron., 2008, 23, $1458-1465$.

18 A. Pasic and H. Koehler, et al., Anal. Bioanal. Chem., 2006, 386, $1293-1302$.

19 R. Dutt-Ballerstadt and C. Evans, et al., Diabetes Technol. Ther., 2008, 10, 453-460.

20 R. B. Thompson, H.-H. Zeng, D. Ohnemus, B. McCranor, M. Cramer and J. Moffett, Methods Enzymol., 2008, 450, 311-336.

21 L. J. McCartney, J. C. Pickup, O. J. Rolinski and D. J. S. Birch, Anal. Biochem., 2001, 292, 216-221.

22 J. S. Marvin and H. W. Hellinga, J. Am. Chem. Soc., 1998, 120, 7-11.

23 L. Tolosa, I. Gryczynski, L. R. Eichhorn, J. D. Dattelbaum, F. N. Castellano, G. Rao and J. R. Lakowicz, Anal. Biochem., 1999, 267, 114-120.

24 L. L. E. Salins, R. A. Ware, M. Ensor and S. Daunert, Anal. Biochem., 2001, 294, 19-26.

25 K. Ye and J. S. Schultz, Anal. Chem., 2003, 75, 3451-3459.

26 M. Fehr, S. Lalonde, I. Lager, M. W. Wolff and W. B. Frommer, J. Biol. Chem., 2003, 278, 19127-19133.

27 X. Ge, L. Tolosa and G. Rao, Anal. Chem., 2004, 76, 1403-1410.

28 K. J. Thomas, D. B. Sherman, T. J. Amiss, S. A. Andaluz and J. B. Pitner, Diabetes Technol. Ther., 2006, 8, 261-268.

29 F. Khan, L. Gnudi and J. C. Pickup, Biochem. Biophys. Res. Commun., 2008, 365, 102-106.

30 F. Khan, T. E. Saxl and J. C. Pickup, Anal. Biochem., 2010, 399, 3943.

31 R. B. Thompson, in Topics in Fluorescence Spectroscopy, ed. J. R. Lakowicz, Plenum Press, New York, 1991, vol. 2, pp. 345-365.

32 D. J. S. Birch, D. McLoskey, A. Sanderson, K. Suhling and A. S. Holmes, J. Fluoresc., 1994, 4, 91-102.

33 J. Schmitt, H. Hess and H. G. Stunnenberg, Mol. Biol. Rep., 1993, 18, 223-230.

34 W. J. O'Hagan, M. McKenna, D. C. Sherrington, O. J. Rolinski and D. J. S. Birch, Meas. Sci. Technol., 2002, 13, 84-91. 